\title{
Overlapping/Multiple Zones of Prostate
}

National Cancer Institute

\section{Source}

National Cancer Institute. Overlapping/Multiple Zones of Prostate. NCI Thesaurus. Code C157522.

A term that indicates involvement of multiple zones of prostate. 